ein solches an die Hand zu geben. Der Beitrag, den die vorliegende Arbeit zu einer Grundlegung einer Wissenschaftssoziologie der Soziologie leistet, liegt in der Entwicklung eines theoretischen wie methodologischen Vokabulars, das auf empirischer Ebene einen soziologischen Zugang zu ihrer Doppelstruktur erlaubt.

\title{
1.2 DIE KONTEXTUIERUNG: DIE DOPPELSTRUKTUR DER SOZIOLOGIE ALS „SOZIOLOGISCHER PROBLEMBÄR“
}

Befragt man die Soziologie selbst, wird deutlich, dass sie ihr Verhältnis zu ihrem Untersuchungsgegenstand überwiegend als Problem wahrnimmt. Man fühlt sich erinnert an den Problembären Bruno, der sich im Jahr 2006 aus seinem Revier im italienischen Naturpark Adamello-Brenta entfernte und über den Edmund Stoiber in einer Rede sagte: „[...] der normal sich verhaltende Bär lebt im Wald, geht niemals - äh - raus und - äh - reißt vielleicht - äh - ein bis zwei Schafe im Jahr. Äh. Wir haben dann einen Unterschied zwischen dem normal sich verhaltenden Bär, dem Schadbär und dem, äh, Problembär." ${ }^{8}$ Zugegeben: Der Vergleich dient mehr der Polemisierung als der akkuraten Beschreibung. Dennoch: Bleibt die Soziologie in ihren geschützten Sphären, ist die Welt eigentlich in Ordnung. Tritt sie jedoch heraus, mögen dies einige mitunter als schädlich für die Disziplin empfinden. Auch wenn dies nicht alle Soziolog(inn)en in dieser Schärfe mittragen, so würden sie den Schritt nach draußen doch zumindest als Problem beschreiben. Dieser Problembär findet in der Literatur drei unterschiedliche Charakterisierungen: 1. Das Theorie-Praxis-Problem der Soziologie; 2. Das Wertproblem der Soziologie und 3. Das Grenzproblem der Soziologie.

\subsubsection{Das Theorie-Praxis-Problem der Soziologie}

Das Problem der Soziologie mit ihrem Untersuchungsgegenstand wird u.a. als Theorie-Praxis-Problem beschrieben. Die betreffenden Autoren trennen konsequent zwischen Theorie einerseits und Praxis andererseits, die in einem dialekta-

8 Der Ausschnitt der Rede Stoibers ist über zahlreiche Kanäle verfügbar. Siehe unter anderem https://rp-online.de/politik/deutschland/zeitlos-schoen-stoibers-satzungetue me_iid-23672143\#12, (Zugriff vom 11.12.18). 
len Verhältnis zueinander stehen ${ }^{9}$ (Kellermann, 1980; Luhmann, 1981; Hülsdünker, 1983; Kaesler, 1984; Mayntz et al., 1998; Kühl, 2003a; Blättel-Mink et al., 2004b; Kurtz, 2007; Voruba, 2017; Offe, 1982; Beck, 2015). ${ }^{10}$ In der Literatur findet sich das Theorie-Praxis-Problem in folgenden Ausprägungen:

- Mangelnde Anwendung soziologischen Wissens in der Praxis

- Mangelnde Professionalisierung der Soziologie

- Mangelnde Legitimität der Soziologie, die Praxis zu kritisieren

\section{Mangelnde Anwendung soziologischen Wissens in der Praxis}

Grundlegend für die Problematisierung der mangelnden Anwendung soziologischen Wissens ist die Trennung zwischen einem „Innen“, der Soziologie, und einem „Außen“, der Praxis. Diese Trennung ist eng mit einer Rhetorik der Grundlagen- bzw. der Anwendungsorientierung verbunden. Damit verknüpft ist wiederum die Vorstellung von externen „Adressaten“ von bzw. „Interessenten“ (Beck, 2015, S. 18) an soziologischer Forschung, die im Zweifelsfall Einfluss auf die Fragestellungen der Wissenschaft nehmen (Kellermann, 1980; Nowotny et al., 1985; Whitley, 1985; Hülsdünker, 1983; Jansen-Schulze, 1997; Clemens, 2001; Latniak et al., 2004; Lengwiler, 2005; Opp, 2005).

Der Problembär liegt in dieser Betrachtungsweise in der soziologischen Wissensproduktion selbst. Soziologisches Wissen sträubt sich quasi gegen seine praktische Anwendung. Es kann die Erwartungen der Praxis nicht erfüllen (Kühl, 2003a). So wird dieses Wissen von der Gesellschaft nicht immer als relevant anerkannt, da keine allgemeingültigen Kriterien von Nützlichkeit bzw. Brauchbarkeit existieren. Zudem spielen unterschiedliche Zeithorizonte und Logiken von Wissenschaft und Praxis genauso eine Rolle wie verschiedene Erwartungen und Sprachen (Albrecht, 1982). Beck konstatiert, dass die Soziologie letztlich die Bedürfnisse derer ignoriert, für die das Wissen gemacht wird (Beck, 1982a). Sie täte deshalb gut daran, die Praxis ernst zu nehmen und nicht wie ein Missionar auf eine bereits missionierte Gesellschaft herunterzublicken (Beck et al., 1989a; Beck et al., 1989b). Ebenso muss ein Problem zunächst als gesellschaftlich relevant markiert werden, um außerhalb der Wissenschaft überhaupt

9 Referenzen der Wissenschaftsforschung zu einer Trennung zwischen Theorie und Praxis siehe unter anderem in Bush, 1960; Stokes, 1997; Latour, 1999; Kaldewey, 2013.

10 Siehe dazu auch den Sonderband der Sozialen Welt, Soziologie und Praxis (Beck, 1982b). 
wahrgenommen zu werden (Nowotny, 1975). ${ }^{11}$ Nowotny nutzt diesbezüglich den Begriff der „Relevierung sozialwissenschaftlichen Wissens“ (Nowotny, 1975, S. 451).

Für Wingens und Fuchs (1989) ist nicht die Frage der fehlenden Relevanz von Wissen die Krux, sondern die mangelnde theoretische Konzeptualisierung des Begriffs der „Verwendung soziologischen Wissen[s]“ (Wingens et al., 1989, S. 210). Denn bereits diese Form der Zuordnung ist auf die Demarkation unterschiedlicher Wissensarten, nämlich soziologischen Wissens und nicht soziologischen Wissens, angewiesen.

\section{Mangelnde Professionalisierung der Soziologie}

Hinter der mangelnden Professionalisierung als Ursache für das Theorie-PraxisProblem der Soziologie steht, dass der Gegenstand der Soziologie unklar ist. Die Gesellschaft ist kein abgegrenztes Feld wie die Wirtschaft oder die Politik (Kühl, 2003b). Unklar ist beispielsweise, was überhaupt als „,soziologisch“ verstanden werden kann (Alemann, 2002). Somit existiert auch kein klares Berufsbild außerhalb der Universitäten; Klient(inn)en und Aufgaben bleiben aus professionssoziologischer Sicht eher vage (Breger, 2007). So ist die Soziologie im Gegensatz zu anderen Disziplinen nicht gleichzeitig eine auf Beratung ausgerichtete Profession, im Studium werden keine Voraussetzungen für diese Form der Praxisorientierung geschaffen (Alemann, 2002). Vermittelt würden nach Kromrey (2007) vielmehr „Schlüsselqualifikationen“ bzw. „fachübergreifende“ oder „Querschnittsqualifikationen“ (Kromrey, 2007, S. 3), die die Studierenden dann auch in der Praxis nutzen könnten. ${ }^{12}$ Das Berufsfeld der „Soziologischen Beratung“ (Blättel-Mink et al., 2004a) wird demnach von vielen Soziologinnen und Soziologen eher zufällig entdeckt als bewusst gesucht (Alemann, 2004, S. 43 ff.). Die mangelnde Professionalisierung äußert sich deshalb auch in einer Kluft zwischen soziologischer Beratung und akademischer Soziologie: Beklagt wird der geringe Rückhalt in der Disziplin (Alemann, 2002) wie auch die fehlende Rückbindung der praktischen Erfahrungen an den soziologischen Diskurs.

Ein weiteres Problem der Professionalisierung der Soziologie liegt in der schwierigen Abgrenzung soziologischen Wissens von Alltagswissen. So attestiert Zimenkova (2007) der Soziologie ein „Sprachproblem“ (Zimenkova, 2007,

11 Siehe dazu auch Nowotnys Ausführungen zu „socially robust knowledge“ (Nowotny, 2003).

12 Hier werden beispielsweise Faktoren aufgeführt wie: organisieren, planen, analytisch denken oder zielorientiert arbeiten. Weniger wichtig erscheinen Aspekte wie soziologische Theorie oder wissenschaftliches Schreiben (Kromrey, 2007, S. 5). 
S. 300). Dieses entsteht aus einem Abgrenzungsmechanismus der Disziplin gegenüber der Praxis, um dieser ihre auf Wissenschaft ausgerichtete Exklusivität und Professionalität zu demonstrieren. Die Folge ist, dass die Soziologie für ein außerwissenschaftliches Publikum nicht mehr zu verstehen ist. Lau und Beck argumentieren, dass soziologisches Wissen dann am erfolgreichsten anwendbar ist, wenn es sich seiner "Identität“ entledigt und im Praxiskontext nicht mehr als solches zu erkennen ist (Beck et al., 1989a, S. 11 f.). Die Soziologie müsste also im Zuge ihrer Professionalisierung ihr Abgrenzungsmerkmal, nämlich ihre soziologische Sprache, aufgeben, was letztlich eine paradoxe Entwicklung zur Folge hätte: eine Entsoziologisierung der Soziologie.

\section{Mangelnde Legitimität der Soziologie, die Praxis zu kritisieren}

Hier steht im Fokus des Theorie-Praxis-Problems die Frage, ob sich die Soziologie überhaupt als Kritikerin der Praxis gerieren kann (Wehling, 2014; Bröckling, 2013). Der Begriff der Praxis wird neu definiert. Praxis wird nicht als außerwissenschaftliche Praxis definiert, sondern der Begriff bezeichnet eine Praxis der Soziologie, die sich nicht sieht als „,unversöhnliche Kritiker[in] einer Gegenwart, die ihre stabilisierenden Institutionen und verbindlichen Orientierungen verloren" hat (Bröckling, 2013, S. 309). In ähnlicher Weise, wie bereits Beck und Bonß (1989a; 1989b) konstatieren, würde die Soziologie nämlich dadurch „Attitüden des Besserwissens" (Luhmann, 1991a, S. 148) einnehmen und der Welt oder „den Leuten“ (Voruba, 2017, S. 179) quasi sagen, wie sie zu leben hätten. Anders verhält es sich mit einer Konzeptionierung von Praxis, die soziologische Praxis in einen Gegensatz zu soziologischer Theorie stellt (Voruba, 2017). Im Zentrum steht hier die Frage, wie die Soziologie als Praxis Kritik an gesellschaftlichen Verhältnissen üben kann, ohne ihr Selbstverständnis als Wissenschaft aufzugeben (Boltanski et al., 1999; Boltanski et al., 2007; Boltanski, 2010; Bröckling, 2013; Voruba, 2017). Die Soziologie wechselt von einer Beobachtung der ersten in eine Beobachtung der zweiten Ordnung (Luhmann, 1991a). In dieser Lesart interveniert die Soziologie also nicht mehr direkt in die Gesellschaft:

„Die Soziologie betreibt hier nicht Kritik, sondern beobachtet Kritik. [...] Es ist keineswegs ein Rückzug, das soziologische Engagement in Sachen Kritik von Betreiben auf Beobachten umzustellen. Vielmehr handelt es sich um die einzige Möglichkeit, das kritische Potenzial der diversen Spielarten kritisierender Philosophie und Soziologie zu bewahren." (Voruba, 2017, S. 177) 
Kritische Soziologie wird also zu einer Soziologie der Kritik. Sie macht sich keinerlei Überheblichkeit schuldig, da sie nicht selbst die Praxis kritisiert, ihr Wissen einbringt oder Lösungen vorschlägt. Sie beschreibt lediglich, was sie an Kritik innerhalb der Gesellschaft beobachtet, und definiert so Soziologie nicht als Theorie, sondern als Praxis (Voruba, 2017).

\subsubsection{Das Wertproblem der Soziologie}

Neben dem Theorie-Praxis-Problem ist das Wertproblem der Soziologie die zweite Art, auf die das Verhältnis der Soziologie zu ihrem Untersuchungsgegenstand problematisiert wird. Hier geht es weniger um eine mangelnde Anwendung oder Professionalisierung der Soziologie als um die normativen Vorannahmen der Forschenden im Forschungsprozess.

„[So steht die] Soziologie [...] in einem dauernden Spannungsverhältnis zur eigenen Alltagswahrnehmung und zu verinnerlichten Wertüberzeugungen. Man muss sich sozusagen persönlich revidieren. Das muss ich z.B. als Geograf nicht.“ (M. Rainer Lepsius in Lepsius et al., 2008, S. 46 f.)

Das Wertproblem der Soziologie nahm und nimmt in soziologischen Selbstreflexionen viel Raum ein (Weber, 1988[1922]; Beck, 1974; Topitsch, 1965; Habermas, 1963; Myrdal, 1971; Myrdal et al., 1965; Habermas, 1982; Prewitt, 2005; Schurz et al., 2013). ${ }^{13}$ Die bereits von Max Weber formulierte Prämisse der „Wertbeziehung“" ${ }^{14}$ der Forschung führt letztlich zu dem Schluss, dass professio-

13 Wissenschaft und Werte sind auch in der Wissenschaftsforschung besonders stark reflektiert worden. Siehe dazu u.a. Kitcher, 2001; Merton, 1973; Visvanathan, 1997; Mulkay, 1976; Keller, 1985; Longino, 1990.

14 Obwohl Weber für eine Trennung zwischen „Sein“ und „Sollen“ plädiert, bleibt er bei der Definition von Wertbeziehung selbst zurückhaltend und geht letztlich nur auf die Wertbeziehung als wissenschaftliches Interesse ein: „Ueber die Bedeutung des Ausdruckes ,Wertbeziehung' muß ich mich auf eigene frühere Aeußerungen und vor allem auf die bekannten Arbeiten von H. Rickert beziehen. Es wäre unmöglich, das hier nochmals vorzutragen. Es sei daher nur daran erinnert, daß der Ausdruck, Wertbeziehung ' lediglich die philosophische Deutung desjenigen spezifisch wissenschaftlichen ,Interesses ' meint, welches die Auslese und Formung des Objektes einer empirischen Untersuchung beherrscht.“ (Weber, 1988[1922], S. 511, Hervorhebung der Autorin). Obwohl Weber hier also interessanterweise die individuellen Interessen ausklammert, wird sein Ausdruck der „Wertbeziehung“ meist anders verwendet. 
nelle Soziolog(inn)en genau zwischen „Sein“ und „Sollen“ trennen sollten (Weber, 1988[1922]). Soziologische Wissensproduktion muss sorgsam von bestehenden Wertvorstellungen der Forschenden unterschieden werden, um nicht als Alltagsmeinung daherzukommen. Bourdieu ruft zu einer „epistemologischen Wachsamkeit“ (Bourdieu et al., 1991, S. 15) auf. Die Soziologie als Wissenschaft habe vor allem die Konstruktion eines Forschungsobjekts zu leisten, das von Ordnungsvorstellungen, Fragestellungen und Problemformulierungen eines Alltagsverständnisses abgelöst sei. Nur so laufe sie nicht Gefahr, aus dem politischen Raum stammende Problemformulierungen unkritisch aufzugreifen. Andernfalls drohe die Soziologie, in „common-sense-Problemformulierungen zu verharren [und] über ,Betroffenheits'-Soziologien oder technokratische Praxisanweisungen" (Bourdieu et al., 1991, S. X) nicht hinauszukommen. Wissenssoziologisch gewendet bewegt sich also das Individuum in einem Spannungsfeld zwischen seinem wissenschaftlichen Arbeiten und der eigenen Alltagswelt (Berger et al., 1991[1966], S. 37). Soziologie ist dementsprechend einem „Problem der Ideologie“ (Korte, 2011, S. 15) ausgesetzt. Eine Soziologie der Kritik wie in 1.2.1 beschrieben wiederum umgeht dieses Problem, zumindest semantisch, weil hier

„dem Soziologen durch Übernahme des Gesichtspunkts des Akteurs möglich [wird], einen normativen Blick auf die Welt zu werfen, ohne daß dieser Blick durch persönliche Optionen (gebunden z.B. an eine spezifische kulturelle Zugehörigkeit, ein politisches oder religiöses Engagement) oder durch den Rückgriff auf eine inhaltliche Moralphilosophie (wie den Utilitarismus) geleitet ist“" (Boltanski, 2010, S. 57).

\subsubsection{Das Grenzproblem der Soziologie}

Wie wir in den vorhergehenden Abschnitten gesehen haben, lässt sich das Verhältnis der Soziologie zu ihrem Untersuchungsgegenstand unterschiedlich spezifizieren. Zum einen geht es um die Trennung zwischen der Theorie und der Praxis als zwei unterschiedlichen Sphären, die ein „Innen“ (die Soziologie) und ein „Außen“ (die Praxis) definieren. Bei der Konzeption einer Soziologie der Kritik wird die Praxis wiederum in die Soziologie hineingeholt; es wird versucht, das „Außen“ der Soziologie möglichst vom Interventionspotenzial der Soziologie abzukoppeln. Eine zweite, eng damit verbundene Wahrnehmung ist die, der Soziologie ein Wertproblem zu bescheinigen. Auch hier geht es um die Definition eines „Innen“ und eines „Außen“, wobei der Fokus in diesem Zusammenhang insbesondere auf dem normativen Gehalt soziologischer Wissensproduktion liegt, der sich (unerlaubterweise) in die wissenschaftliche Analyse hineinmogelt. 
Die Trennung, die hier vorgenommen wird, konstruiert also ein „Innen“, nämlich den Forschenden mit seinen Werturteilen, und ein „Außen“, nämlich den Untersuchungsgegenstand, der möglichst wenig von dem „Innen“ berührt werden sollte.

In der klassischen Wissenschaftsforschung ist diese Trennung nichts Besonderes (Kaldewey, 2013). Es ist eine Besonderheit der Soziologie, dass eine einfache Referenzierung von „Innen“ (Theorie) und „Außen“ (Praxis) nicht gut funktioniert. ${ }^{15}$ Die These dieser Arbeit ist, dass die Soziologie weder ein Theorie-Praxis-Problem noch ein Wertproblem hat. Vielmehr ergeben sich diese Diagnosen aus einem fundamentaleren Problem, das in dieser Arbeit als Grenzproblem spezifiziert wird. Nun mag in dieser These zunächst nichts Überraschendes liegen. Im Gegenteil: Es existieren eine Reihe soziologischer Zugänge, die bereits in der einen oder anderen Weise Grenzen in der Soziologie zum Thema gemacht haben.

Aus einer wissenssoziologischen Perspektive wird zunächst von diversen Autorinnen und Autoren moniert, dass die Soziologie ihre Wissensbestände gegenüber Alltagswissen abzugrenzen und als soziologisches Wissen zu markieren hat. Bereits hier ist nämlich die Demarkation nicht klar, da soziologisches Wissen sich quasi als Parasit auf lebensweltliche Wissensbestände aufsetzt, diese analysiert, klassifiziert und „soziologisiert“. Gans (1989) argumentiert in diesem Zusammenhang, dass alle Menschen als Laiensoziologen zu betrachten sind, da sie spezifische Deutungen des Sozialen vornehmen. Zum einen kann dies zu Konflikten zwischen wissenschaftlichen Konzeptionen und „lay images“ (Lammers, 1974, S. 125) - also alltagsgebundenen Hintergrundannahmen über das Soziale, führen. Die Sozialwissenschaften sind durch diese lay images schnell dem Vorwurf der Überflüssigkeit ausgesetzt: ,[I]t tells you what you knew already anyhow.“ (Lammers, 1974, S. 137) So argumentiert Habermas, dass der Objektbereich der Soziologie bereits symbolisch vorstrukturiert und vorwissenschaftlich konstituiert ist und dass die Soziologie auf den „Eigensinn“ (Habermas, 1982, S. 549) des Objektbereichs reagieren muss. Zum anderen verfügen professionalisierte Soziolog(inn)en über eigene lay images (Lammers, 1974). Diese Annahmen gehen unweigerlich in jegliche Deutung des Sozialen ein und mithin auch in jede Form der professionalisierten Soziologie (Habermas, 1982; Gans, 1989). Die Soziologie produziert ja, wie eben gehört, kein der Gesellschaft

15 Hier schließt sich die Arbeit auch an Kaldeweys These an, der diese einfache Referenzierung ablehnt und Autonomiediskurse wie auch Praxisdiskurse als „konstitutiv für das [ansieht], was wir heute Wissenschaft nennen“ (Kaldewey, 2013, S. 26). 
fremdes Wissen, sondern ist selbst auf eben dieses Wissen angewiesen (Giddens et al., 1982; Habermas, 1982; Elias, 1983).

Die Soziologie und ihr Untersuchungsgegenstand lassen sich deshalb nicht klinisch genau voneinander trennen. Sie reagiert auf ihren Untersuchungsgegenstand genauso wie dieser wiederum auf die Soziologie reagiert. Giddens markiert hier den Unterschied zu den Naturwissenschaften, den er als doppelte Hermeneutik der Sozialwissenschaften bezeichnet (Giddens et al., 1982; Giddens, 1979; Giddens, 1984):

„There are no universal laws in the social sciences, and there will not be any - not, first and foremost, because methods of empirical testing and validation are somehow inadequate but because, as I have pointed out, the causal conditions involved in generalizations about human social conduct are inherently unstable in respect of the very knowledge (or beliefs) that actors have about the circumstances of their own action. The so-called, selffulfilling prophecy', of which Merton and others have written, is a special case of a much more generic phenomenon in the social sciences. This is a mutual interpretative interplay between social science and those whose activities compose its subject matter - a,double hermeneutic'. The theories and findings of the social sciences cannot be kept wholly separate from the universe of meaning and action which they are about. But, for their part, lay actors are social theorists, whose theories help to constitute the activities and institutions that are the object of study of specialized social observers or social scientists. There is no clear dividing line between informed sociological reflection carried on by lay actors and similar endeavours on the part of specialists. I do not want to deny that there are dividing lines, but they are inevitably fuzzy, and social scientists have no absolute monopoly either upon innovative theories or upon empirical investigations of what they study." (Giddens, 1984, S. xxxii f., Hervorhebung der Autorin)

Die Soziologie zeichnet sich also durch eine Doppelstruktur aus, indem sie einerseits auf Wissensbeständen des Sozialen aufbaut, von denen sie sich andererseits distanzieren muss. Ihre Wissensbestände sind nicht eindeutig von denen ihres Untersuchungsgegenstandes zu trennen, vielmehr reagiert die Gesellschaft auf das, was die Soziologie sagt, mit eigenen Theorien und Annahmen und hält diese ja bereits vor einer soziologischen Analyse bereit. Die Grenzen sind dementsprechend unscharf und die Sozialwissenschaften, insbesondere die Soziologie, entbehren eines Deutungsmonopols. Aufgrund dieser Doppelstruktur kann ihr die Distanzierungsleistung nie ganz gelingen. Daraus ergibt sich das bereits als Wertproblem der Soziologie markierte Element soziologischer Forschung: 
„The fact that the ,findings' of the social sciences can be taken up by those to whose behaviour they refer is not a phenomenon that can, or should, be marginalised, but is integral to their very nature: It is the hinge connecting two possible modes in which the social sciences connect to their involvement in society itself: as contributing to forms of exploitative domination, or as promoting emancipation.“ (Giddens et al., 1982, S. 14)

Anders gesagt: Um einen ihr fremden Standpunkt darzustellen, muss die Soziologie also zunächst ihren eigenen Standpunkt klären. Diese Notwendigkeit erfordert zugleich eine Grenzziehung zwischen einem „Innen“ und „Außen“ ihrer Beobachtung. Nun ist es nur plausibel zu entgegnen, dass auch die Naturwissenschaften auf eine Grenzziehung eines „Innen“ und „Außen“ angewiesen sind (Kaldewey, 2013, S. 26 ff.). Das ist im Prinzip natürlich richtig und konsequent, erkennte man das nicht an, würde man sich gegen die neueren Erkenntnisse der Wissenschaftsforschung selbst stellen. Dennoch ist die These, dass für die Sozialwissenschaften, insbesondere die Soziologie, aufgrund der Stellung zu ihrem Untersuchungsgegenstand ein besonderes Grenzproblem besteht.

In der soziologischen Literatur wird diese besondere Stellung in Kontrast zu den Naturwissenschaften reflektiert. Habermas stellt sich in diesem Zusammenhang die Frage, „ob durch den Umstand, dass der Mensch [...] zugleich Subjekt und Objekt der Wissenschaft ist, ein prinzipieller Unterschied im Vergleich zur Situation der Naturwissenschaften bedingt ist" (Habermas, 1982, S. 546). Für Habermas macht es einen Unterschied: Der Sozialwissenschaftler ist Laie und Wissenschaftler zugleich. Er bezieht sich auf das vortheoretische Wissen eines sozialisierten Erwachsenen und dessen Lebenswelt. Hinzu kommt, dass im Vergleich mit den Naturwissenschaften wesentlich mehr lay images zu Thematiken der Sozialwissenschaften in der Gesellschaft bestehen. Soziologie ist in der Hinsicht deshalb leichter angreifbar als die Naturwissenschaften.

So ist es den Naturwissenschaften erfolgreicher als den Sozialwissenschaften gelungen, institutionelle Grenzarbeit zwischen „Grundlagenforschung“ und ,angewandter Forschung“ zu betreiben und Nachweise ihrer „Nützlichkeit“ zu erbringen. Ein gutes Beispiel ist in diesem Zusammenhang die Einführung des öffentlichen Experiments im 17. Jahrhundert. Es war ein physischer und sozialer Raum, in dem Experimente ausgeführt und beobachtet werden konnten (Shapin et al., 2011). Wissenschaftliche Fakten wurden vor den Augen der Zuschauer geschaffen und validiert. Im Gegensatz dazu war das Labor zuvor ein privater Raum gewesen, ,inhabitated by secretists“ (Shapin et al., 2011, S. 335). Objekt und Subjekt der Forschung konnten voneinander getrennt werden, genauso wie die wissenschaftliche Beschäftigung mit der Natur von der Beschäftigung mit den ,human affairs“, die untrennbar mit Staat und Kirche verstrickt waren 
(Shapin et al., 2011). So konnte das Bild einer Wissenschaft entstehen, in dem die Naturwissenschaften auf Basis des Experiments die Natur beherrschen und Vorhersagen über diese treffen können. Aus dem Eingangszitat geht hervor, wie sehr sich die Soziologie etwas Vergleichbares bereits in ihren Anfängen, beeinflusst von positivistischen Strömungen, gewünscht hat. Um Missverständnisse auszuräumen: Es geht hier nicht darum, ein essenzialistisches Wissenschaftsbild der Naturwissenschaften als „harte Wissenschaften“ vs. die Sozialwissenschaften als ,weiche Wissenschaften“ zu erzeugen. Vielmehr haben die Naturwissenschaften es (in einem schon fast als „Imagekampagne“ zu bezeichnenden Unterfangen) wesentlich geschickter geschafft, die Grenzen zwischen sich und ihren Untersuchungsgegenständen zu ziehen, als dies der Soziologie gelungen ist. ${ }^{16}$

Teile der Wissenschaftsforschung gehen von einer Co-Produktion (Jasanoff, 2004b) oder Co-Evolution (Nowotny et al., 2001) von Sozialwissenschaften und Gesellschaft aus (Porter, 1995; Desroisères, 1994; Rueschemeyer et al., 1996; Scott, 1998; Nowotny, 1994; Wagner, 1990; Boltanski, 2014). Aufgrund dieser Co-Produktion bzw. -Evolution scheint das „Verhältnis der Sozialwissenschaften zur Gesellschaft anders strukturiert zu sein [...] als dasjenige zwischen Naturwissenschaften und Gesellschaft" (Reinhart, 2016, S. 162). Die Soziologie verfügt über kein Labor, in dem sie ihr Wissen transparent und als sogenannte Fakten nachweisbar produzieren könnte. Dagegen stehen zum einen ethische Bedenken - zum anderen wäre es schlichtweg nicht möglich, die Gesellschaft in all ihrer Komplexität in einem Labor nachzustellen. ${ }^{17}$ So argumentiert Luhmann,

16 Ein Großteil der Science and Technology Studies hat sich kritisch mit der Produktion naturwissenschaftlicher Fakten auseinandergesetzt (Knorr-Cetina, 1981; Latour, 1988; Latour, 1993; Latour, 1999; Fuller, 1991; Knorr-Cetina, 1999; Jasanoff, 1995b; Jasanoff, 2004a; Jasanoff, 2004c). Diese Literaturangaben stellen einen kleinen Ausschnitt der wichtigsten Referenzen der Science and Technology Studies zu dem Thema dar. Die relevante Literatur vollständig anzugeben, ist in diesem Kontext nicht machbar, aber auch nicht notwendig. Gute Einblicke in die Thematik geben unter anderem folgende Überblickswerke: Jasanoff, 1995a; Hackett et al., 2008; Sismondo, 2010.

17 Wobei natürlich auch hier angemerkt sei, dass Experimente sich in den Sozialwissenschaften, insbesondere in der Psychologie und neuerdings auch der Ökonomie steigender Beliebtheit erfreuen. Somit können sie ähnlich wie die Naturwissenschaften ein Bild von sich als Wissenschaft erzeugen, die in der Lage ist, objektive Fakten herzustellen. Die methodologische Kritik an solchen Experimenten spielt laut Fuller (1991) eine untergeordnete Rolle: ,[...] these sciences seem to be epistemologically sound only because we have learned to turn a blind eye to the many times when avowed methodology and actual practice diverge.“ (Fuller, 1991, S. 308) 
dass die Soziologie eben gerade über kein Labor wie die Naturwissenschaften verfügt. ${ }^{18}$ Sie kann ihren Gegenstand nicht einfach externalisieren, da sie immer interne Beobachterin des Systems ist. Dementsprechend gelten die klassischen Subjekt-Objekt-Unterscheidungen nicht, da die Soziologie ja Teil des Objekts ist, das sie beschreibt. Sie hat also ein ontologisches Problem. Denn obwohl sie beobachtet, kann sie sich nicht als unabhängige Reflexionsinstanz begreifen (Luhmann, 1993). So also konstruiert die Soziologie einen Gegenstand, dessen Teil sie selbst ist. Das Außen ist größer als das Innen. Ihr ontologisches Problem wird zu einem autologischen Zustand erhoben: Die Soziologie kann sich nur innerhalb der Gesellschaft beschreiben und beschreibt damit auch immer sich selbst. In Hinblick auf die Entstehung eines soziologischen Diskurszusammenhangs argumentiert Beetz, dass sich die Disziplin bei ihrer Entstehung um eine doppelte Leitfrage gruppierte, und zwar ,wie man zum Gegenstand des Fachs stehe, sowie welches die Rolle der Soziologie innerhalb der Gesellschaft sein solle bzw. sein könne“ (Beetz, 2014, S. 21). Die erste Frage ordnet er dem ontologischen Diskussionszusammenhang zu, die zweite dem autologischen.

Betrachtet man die Doppelstruktur der Soziologie systemtheoretisch, befindet sie sich also in einem Spannungsverhältnis zwischen Autologie und Ontologie, in dem es ihr niemals möglich ist, aus der Gesellschaft herauszutreten. Vielmehr sind ihr Reflexionen in Hinblick auf ihr Verhältnis zum eigenen Untersuchungsgegenstand inhärent. ${ }^{19}$

18 Dennoch verfügt die Soziologie in gewissem Maß über eine Labortradition. So verweist die Chicago School auf die Stadt als ein Labor (Gross et al., 2005). Die Rahmenbedingungen werden durch die Gesellschaft selbst festgelegt, sind also bereits von der Gesellschaft „,arrangiert“ (Gross et al., 2005, S. 62). Das unterscheidet sie im Wesentlichen von den Vorstellungen naturwissenschaftlicher Laborexperimente. Einerseits entziehen sich soziale Beziehungen Kausalgesetzen, andererseits lassen sie sich nicht künstlich wie im klassischen Labor modellieren, da dies zudem ethisch nicht zu verantworten wäre. Ziel der Soziolog(inn)en der Chicago School war es, die Gesellschaft besser zu verstehen und daraus nutzbares Wissen abzuleiten. So wurden bspw. Universitätsgelehrte dazu angehalten, längere Zeit in sozial schwachen Gegenden zu wohnen, um die Menschen dort aufzuklären und zu mobilisieren (Gross et al., 2005). Später jedoch distanzierte sich die Chicago School von einer solchen Form des sozialen Engagements zugunsten ihrer Akademisierung (Abbott, 1999, S. 31).

19 Damit ist ein Paradoxon bezeichnet, auf das an dieser Stelle hingewiesen sei, das jedoch hier nicht vollständig aufgelöst werden kann. Denn einerseits ist die Soziologie Teil ihres eigenen Untersuchungsgegenstandes, der Gesellschaft. Andererseits konstruiert sie eben diesen Gegenstand erst selbst, wenn sie als Disziplin antritt, Beobach- 
Wiederum anders begreift Cole (1994) das Grenzproblem der Soziologie im Vergleich zu den Naturwissenschaften. So argumentiert Cole, dass die Soziologie aufgrund der Wandlungsfähigkeit ihres Gegenstandes und der Teilnahme am Gegenstand im Gegensatz zu den Naturwissenschaften selbst keinen disziplinären Kern ausgebildet hat wie beispielsweise die Naturwissenschaften. ${ }^{20}$ Auch andere Autoren sprechen von einer „Fragmentierung“ (Whitley, 1984a; Whitley, 1984b) oder polyparadigmatischen (Lammers, 1974) Ausrichtung der Sozialwissenschaften.

„Sociology's claim of being a general social science is thus inseparable from its varied relations to other disciplines and domains of knowledge. As a consequence [...], sociology has been a discipline with a high degree of plasticity. What sociologists collectively produce tends to have a low level of cognitive and professional codification, a high degree of pluralism, dispersion, and context dependency, and a rather volatile intellectual status.“ (Heilbron, 2011, S. 181)

Auch wenn verschiedene soziologische Zugänge das Grenzproblem der Soziologie unterschiedlich spezifizieren, so ist ihnen doch gemein, dass sich das Grenzproblem der Soziologie auf eine ihr inhärente Doppelstruktur zuspitzen lässt, in dem die Zuordnungen von „Innen“ und „Außen“ wesentlich weniger offensichtlich sind, als dies bei den Naturwissenschaften der Fall ist (siehe Abbildung 1).

tungen über das Soziale anzufertigen. Auch in der soziologischen Literatur wird dieses Paradoxon nicht vollständig aufgelöst - so greifen bspw. soziologische Standardwerke auf beide Begriffskategorien zurück, wenn es einerseits um die Beschreibung von Soziologie in der Gesellschaft und andererseits um die Beschreibung analytischer Kategorien in der Soziologie (unterschiedliche Gesellschaftsbegriffe und Formen, Gesellschaft zu analysieren) geht (Korte, 2011; Giddens et al., 2009a; Fuchs-Heinritz et al., 2011; König, 1958). Es kann in dieser Arbeit jedoch nicht um die Entwicklung eines konsistenten Gesellschaftsbegriffs gehen - dies kann und soll im Kontext dieser Arbeit nicht geleistet werden.

20 Siehe dazu auch Stinchcombe, 1994. 


\section{Abbildung 1: Die Doppelstruktur der Soziologie}

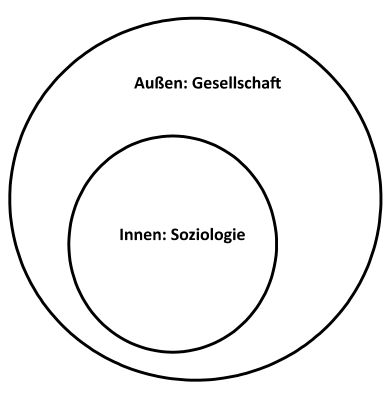

Doppelstruktur der Soziologie nach Luhmann

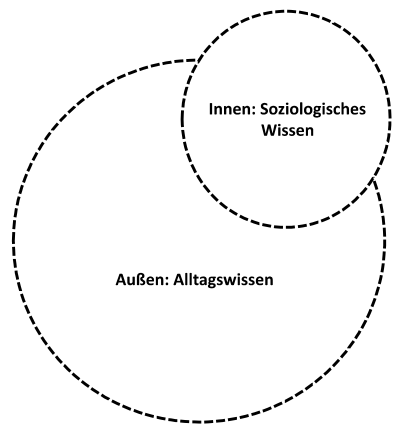

Doppelstruktur der Soziologie nach Habermas und Giddens

Wesentlich für eine solche Innen-Außen-Unterscheidung ist letztlich die Hintergrundannahme, dass die Soziologie ihren Gegenstand objektiviert, also fassbar macht, und dass sie sich selbst von ihm distanziert. Grenzdebatten sind also der Soziologie inhärent. Die Soziologie externalisiert die Zugehörigkeit zu ihrem Gegenstand und setzt Objektivierungstechniken ein: So sollen auch wissenschaftliche Texte „keine zu deutlichen Spuren der Subjektivität des Autors und seines sozialen Kontexts hinterlassen“ (Burkart, 2003, Sp. 6).

Das hier beschriebene Grenzproblem der Soziologie ist eines der konstitutiven Aspekte der Soziologie, mit der sie seit ihrer Entstehung zu kämpfen hat und über das bis heute kein Konsens besteht. Letztlich befindet sie sich in einem Dilemma: Gilt ihre Distanzierungsleistung von Alltagsdiskursen als zu schwach, werden Zweifel an ihrer Zuordnung zur Wissenschaft wach. Gilt ihre Distanzierungsleistung als zu stark, wird wiederum ihre gesellschaftliche Legitimität infrage gestellt. Dennoch hat sie bislang keinen Konsens ausgebildet, wie sie mit ihrem Grenzproblem umgehen soll. Vielmehr erschwert ihr diese Doppelstruktur die Konsensbildung. Die Einigkeit besteht in der Uneinigkeit darüber, oder, wie Kieserling es ausdrückt:

„Daß es der Soziologie nicht leichtfällt, einen Begriff ihrer selbst zu formulieren, ist bekannt. Jeder ausreichend spezifisch formulierende Versuch, der in dieser Richtung gewagt wurde, hat zu Konflikten geführt, und wenn man Konflikte vermeiden will, so wie es in einer jüngeren Generation mit massiven Karriereproblemen ein derzeit mächtiger Trend 
ist, dann muß man auch auf einen Begriff für die Einheit des Faches verzichten.“ (Kieserling, 2004, S. 22 f.)

Ziel der Arbeit ist es nicht, einen einheitlichen Begriff von Soziologie zu entwickeln. Im Gegenteil: Es soll gerade herausgearbeitet werden, wie unterschiedlich das Verhältnis der Soziologie zu ihrem Untersuchungsgegenstand verstanden wird. Dahinter steht die These, dass diese unterschiedlichen Auffassungen Ausdruck einer disziplinären Identitätssuche und eines Selbstverständnisses sind, das unter anderem konstitutiv dafür ist, was unter „guter“ Soziologie verstanden, von der Disziplin als gültiges Wissen anerkannt sowie in interne Debatten aufgenommen wird (Wagner, 1990; Lau, 1984). Denn gerade die Analyse von Grenzdebatten eignet sich letztlich hervorragend, um, im Sinne einer Wissenschaftssoziologie der Soziologie, Einsichten in das Fach selbst zu gewinnen. Denn Grenzen definieren letztlich, was Wissenschaft von Nicht-Wissenschaft unterscheidet:

„Disciplinary boundaries provide the structure needed for a variety of functions, ranging from the allocation of cognitive authority and material resources to the establishment of reliable access to some extra-social reality." (Fuller, 1991, S. 302)

\subsection{DAS DESIDERAT: DIE ANALYSE DER DOPPELSTRUKTUR DER SOZIOLOGIE ALS GRUNDLEGUNG ZU EINER WISSENSCHAFTSSOZIOLOGIE DER SOZIOLOGIE}

So weit, so gut. Wir sehen uns jedoch in dieser Arbeit mit zwei wesentlichen Herausforderungen konfrontiert, die uns in den weiteren Ausführungen beschäftigen werden. Zunächst ergibt sich die Frage, auf welcher Basis das Grenzproblem der Soziologie untersucht werden kann und mit welchen Mitteln? Das Grenzproblem der Soziologie lässt sich in unterschiedlichster Form beschreiben und zuspitzen. Damit sind nicht nur die diversen wissenssoziologischen, wissenschaftsphilosophischen und -historischen Zugänge gemeint, die unter Punkt 1.2 teilweise bereits angerissen wurden. Es geht auch um die Frage, auf welcher Ebene das Grenzproblem begriffen und dementsprechend auch theoretisch und empirisch gefasst werden kann.

Hier kommen wir zum Kernanliegen dieser Arbeit. Denn stellt man die Frage nach dem Verhältnis der Soziologie zu ihrem Untersuchungsgegenstand neu, so kommt man nicht umhin zu überlegen, auf welcher Basis epistemologischer 